\title{
Some consequences of the effective low-energy lagrangian for gravity
}

\author{
A. Dobado and A. López \\ Departamento de Física Teórica \\ Universidad Complutense de Madrid \\ 28040 Madrid, Spain
}

February 1, 2008

\begin{abstract}
We consider the minimal low-energy action for gravity up to six derivatives which is renormalizable at the two-loop level modulo higher derivative corrections. Then we study the classical solutions corresponding to the Schwarzschild and the Robertson-Walker metrics. In the first case we find a singularity close to the gravitational radius and in the second case we find inflationary de Sitter solutions in absence of any matter or cosmological constant.
\end{abstract}

$\mathrm{FT} / \mathrm{UCM} / 7 / 93$ 


\section{Introduction}

Today most of the physicist agree that the issue of quantum gravity is probably the most important open problem in theoretical physics (for a recent review see [1] and references therein). At the present moment it is not clear if the formulation of the quantum theory of gravitation will require going beyond quantum field theory like it happens in string theory or even a deeper modification of quantum mechanics.

Of course this question will not be solved here, but until it is clarified one can adopt a much more modest attitude trying to figure up which could be the low-energy impact of the real theory of gravitation. It is clear that at low energies this theory will lead to the standard Einstein lagrangian. However, this lagrangian will in general be modified by higher-order terms in the sense of the number of derivatives of the space-time metric over the Planck mass scale $M_{P}$. Thus, the Einstein lagrangian with two derivatives and one factor $M_{P}^{2}$ would be the first term, then we would have terms with four derivatives and one $M_{P}^{0}=1$ factor, next we would have six derivative terms with one $M_{P}^{-2}$ factor and so on. The form of all these terms is dictated just by the number of the derivatives and general covariance. Different underlying gravitational theories will predict different values for the adimensional constants affecting these terms, being superstring theories a typical example [2].

Independently of the concrete value of the constants in this low-energy effective lagrangian for gravitation, it is clear that one can use this derivative expansion up to some number of $\partial^{2} / M_{P}^{2}$ powers to predict the qualitative behavior of the modifications to the standard Einstein gravitation theory. However, in order to be completely consistent one should also take into account the effect of the quantum corrections to this effective lagrangian. For example the loops obtained with the Einstein lagrangian produce corrections which are of the order of $\left(\partial^{2} / M_{P}^{2}\right)^{2}$, two-loop diagrams give rise to corrections which are of the order of $\left(\partial^{2} / M_{P}^{2}\right)^{3}$ etc.

This framework for the effective description of the low-energy behavior of gravity has some similitude with that proposed by Weinberg some time ago [3] for the description of the low-energy pion scattering. In that case the effective lagrangian is also the most general lagrangian compatible with the (approximate) chiral symmetry of the strong interactions $S U(2)_{L} \times S U(2)_{R}$ which is supposed to be spontaneously broken to the group $S U(2)_{L+R}$. Pions are understood as the Goldstone bosons associated to this symmetry breaking and this is why they are (nearly) massless. The corresponding fields $\pi_{a}$ are 
just coordinates parametrizing the coset space $S U(2)_{L} \times S U(2)_{R} / S U(2)_{L+R}$. This space is a three sphere i.e. a maximally symmetric space having the chiral group $S U(2)_{L} \times S U(2)_{R}$ as isometry group. At low energies the pion dynamics is controlled by the effective lagrangian

$\mathcal{L}_{e f f}=\frac{1}{2} g_{a b}(\pi) \partial_{\mu} \pi^{a} \partial^{\mu} \pi^{b}+\alpha\left(g_{a b}(\pi) \partial_{\mu} \pi^{a} \partial^{\mu} \pi^{b}\right)^{2}+\beta\left(g_{a b}(\pi) \partial_{\mu} \pi^{a} \partial^{\nu} \pi^{b}\right)^{2}+O\left(\partial^{6}\right)$

where $g_{a b}$ is the metric on the sphere. Here again the quantum corrections to the two derivative term are of the order of $O\left(\partial^{4}\right)$ etc. In principle the constants $\alpha, \beta \ldots$ can be obtained from an underlying theory of the strong interactions like QCD. Nevertheless, even in the case that this theory were not known or the computation of the constants were hard or impossible, the above lagrangian can be very usefully used by obtaining the constants from the experiment and using them to make new predictions. This program was carried out by Gasser and Leutwyler in a remarkable set of papers [4] to the one loop level and ever since this kind of approach to the low-energy hadron interactions has been followed by many particle physicists with great phenomelogical success. More recently, a similar approach has been also applied to the phenomenological description of the unknown symmetry breaking sector of the standard model and in particular to the parametrization of the elastic scattering of the longitudinal components of the weak bosons at the TeV scale [5].

Following this very general line of thinking one could try to make an effective low-energy description of gravity. However, some differences appear when we compare pions or longitudinal components of the weak bosons with gravitons. First we do not have any coset space to make a Goldstone boson like interpretation of gravitons (see [6] for some attempt in this direction). Second, gravity is a gauge theory and we need to go through the standard procedure of quantization of these theories including gauge fixing and ghost terms in the lagrangian to relate properly the ten independent components of the metric tensor with the two physical degrees of freedom of the graviton. In spite of these facts, some analogies still remain with the case of the nonlinear sigma models. For example, as it was mentioned above, the one-loop radiative corrections to the Einstein lagrangian have four derivatives. The divergent terms of these corrections were computed some time ago by 't Hooft and Veltman [7]. Using the background field method and dimensional 
regularization it is possible to find:

$$
\mathcal{L}_{\text {eff }}^{(1)}=\frac{1}{(4 \pi)^{2} \epsilon}\left(\frac{R^{2}}{120}+\frac{7}{20} R^{\alpha \beta} R_{\alpha \beta}\right)
$$

The important point here is that these divergences do not contribute to the $S$ matrix elements (note that they vanish on shell) so that, the Einstein theory of gravitation considered as a standard quantum field theory is oneloop finite. In fact there is a simple argument to see that it must be so. The four-derivative local terms that in principle can be generated by the one loop computation are $R^{2}, R^{\alpha \beta} R_{\alpha \beta}$ and $R_{\gamma \delta}^{\alpha \beta} R_{\alpha \beta}^{\gamma \delta}$. Only the last one does not vanish on shell, but as it is well known the combination $-4 R_{\gamma \delta}^{\alpha \beta} R_{\alpha \beta}^{\gamma \delta}+$ $16 R^{\alpha \beta} R_{\alpha \beta}-4 R^{2}$ is a total derivative in four dimensions related (for Euclidean signature) with the Euler class of the space-time manifold. Thus, modulo a renormalization of this topological term, the one-loop divergences of pure Einstein gravity vanish on shell.

More recently, the two-loop divergent contribution to the effective action for gravity was computed by Goroff and Sagnotti [8]. In this huge computation they were forced to use an special $C$ code since standard algebraic manipulators were unable to work it out completely (see [9] for a previous disscussion about this point). The on-shell contribution to the effective lagrangian coming from the Goroff and Sagnotti computation is given by:

$$
\mathcal{L}_{\text {eff }}^{(2)}=\frac{209}{360(4 \pi)^{3} M_{P}^{2} \epsilon} R_{\gamma \delta}^{\alpha \beta} R_{\sigma \rho}^{\gamma \delta} R_{\alpha \beta}^{\sigma \rho}
$$

Therefore, this remarkable computation showed that the Einstein theory of gravitation is not renormalizable in the common sense. However, one can still consider it as the first term of a derivative expansion by following the philosophy of the phenomenological lagrangians. The new terms that must be added to some given order in the number of derivatives play a double role. First, they have coefficients that carry the information about the underlying theory of gravitation and second, they are also counter terms that absorb the divergences. It could be possible in principle to compute the low-energy action of gravitation in this way up to some number of derivatives i.e. powers of the external momenta, in terms of some renormalized constants that in principle could be obtained from the experiments or observations.. Once they were fitted, this effective action could be used to make new predictions 
Obviously, this approach has many difficult problems that must be solved. First, from the theoretical point of view, the practical computation is very hard. Note that we only know the divergent part of the one and two-loop effective action but we even do not know the one-loop finite part contribution. This kind of contribution is in general non-local since gravitons are massless. Second, we have of course phenomenological problems. As the higher derivative terms are suppressed by powers of $M_{P}$ they become practically unobservable. The only possibility for these terms to be relevant is in physical contexts where the curvature is very large. Typically these situations occur in the regions close to the singularities appearing in the classical solutions of the standard theory and in fact it could be the case that the effective low-energy theory of gravitation completely avoids these singularities solving one of the old problems of the Einstein theory (we will see later that this is not necessarily the case).

Motivated by this interesting possibility we have started the program of studying the effect on the classical solutions of the gravitation theory including the higher local derivative terms that appear in the low-energy effective action for gravity. In fact some of these terms have already been considered in the literature, typically the four derivative ones. However, here we will adopt a different attitude. As the general analysis is in principle very complex we want to select the minimal set of terms that must be added to the standard lagrangian to have a sensible low-energy effective lagrangian. From the Goroff and Sagnotti work we know that, at the six derivatives level, the only term we need to add to get an on-shell finite effective action is that of eq.3. Therefore, we can conclude that the addition of this term to the standard lagrangian produces the minimal consistent effective lowenergy two-loop renomalizable lagrangian in the sense of the effective theories considered in [3] i.e. neglecting higher derivative terms. For the sake of simplicity this will be the only one considered in this note, so that we pass by the more commonly considered in the literature four derivative terms. In this note we will explore the effect of the six derivative term on the important Schwarzschild and Robertson-Walker metrics. 


\section{The equations of motion}

According to the above discussion we will consider the effective action:

$$
\Gamma_{e f f}\left[g_{\alpha \beta}\right]=\int d^{4} x \sqrt{g} \mathcal{L}_{e f f}
$$

where the effective lagrangian is given by:

$$
\mathcal{L}_{\text {eff }}=-\frac{M_{P}^{2}}{16 \pi} R+\frac{\alpha}{M_{P}^{2}} R_{\gamma \delta}^{\alpha \beta} R_{\sigma \rho}^{\gamma \delta} R_{\alpha \beta}^{\sigma \rho}
$$

where $R_{\gamma \delta}^{\alpha \beta}$ is the Riemann tensor, $R$ is the curvature scalar (we will follow the notation convention of the Weinberg's book [10]), $M_{P}$ is the Planck mass and $\alpha$ is an adimensional coupling constant. By applying the standard methods we can obtain the corresponding equation of motion:

$$
\begin{gathered}
\frac{M_{P}^{2}}{16 \pi}\left(R^{\mu \nu}-\frac{1}{2} g^{\mu \nu} R\right)=-\frac{\alpha}{M_{P}^{2}}\left(\frac{1}{2} g^{\mu \nu} R_{\gamma \delta}^{\alpha \beta} R_{\sigma \rho}^{\gamma \delta} R_{\alpha \beta}^{\sigma \rho}\right. \\
\left.-3 R_{\gamma \delta}^{\alpha \mu} R_{\sigma \rho}^{\gamma \delta} R_{\alpha}^{\sigma \rho}{ }_{\alpha}+6\left(R_{\sigma \rho}^{\nu \delta} R^{\sigma \rho \mu \beta}\right)_{; \delta ; \beta}\right)
\end{gathered}
$$

When matter is present, we have to include a new piece in the effective lagrangian $\mathcal{L}_{m}$ and as a consequence of that we must add a new term in the RHS of the above equation of motion which is $-T^{\mu \nu} / 2$ being $T^{\mu \nu}$ the matter energy-momentum tensor. Obviously the Einstein equations of motion are obtained just by taking $\alpha=0$, but for general $\alpha$, the modified equations will produce new effects that will be studied in next section.

\section{The modified Schwarzschild metric:}

The most general form of the static and isotropic metric can be written as:

$$
d \tau^{2}=B(r) d t^{2}-A(r) d r^{2}-r^{2}\left(d \theta^{2}+\sin ^{2} \theta d \phi^{2}\right)
$$

By inserting this metric in the eq.6, we can find the equations to be satisfied by the $A(r)$ and $B(r)$ functions. For the case $\alpha=0$, these equations were solved by Schwarzschild in 1916 for the case of a flat spatial infinity:

$$
\begin{gathered}
B(r)=1-\frac{2 M}{r M_{P}^{2}} \\
A(r)=1 / B(r)
\end{gathered}
$$


being $M$ the total mass of the body producing the gravitational field. For general $\alpha$, the task of finding the corresponding solutions is very hard. However, following the phylosophy of the effective lagrangians, one could try to find at least the first coefficients of the large distance expansion of the $A(r)$ and $B(r)$ functions:

$$
\begin{aligned}
& A(r)=\sum_{n=0}^{\infty} a_{n}\left(\frac{L_{p}}{r}\right)^{n} \\
& B(r)=\sum_{n=0}^{\infty} b_{n}\left(\frac{L_{p}}{r}\right)^{n}
\end{aligned}
$$

where $L_{p}$ is the Planck lenght $\left(L_{p}=1 / M_{P}\right)$ and $a_{n}$ and $b_{n}$ are adimensional coefficients with $a_{0}=b_{0}=1$. Introducing these expansions into the equations of motion, we find that the first correction to the Schwarzschild solution appears to be of the order of $\left(L_{p} / r\right)^{6}$. To find these corrections, we can introduce the Schwarzschild solutions in the RHS of the equation of motion and a completely general static, isotropic metric in the LHS. With the help of a REDUCE code we find the following equations:

$$
\begin{gathered}
-\frac{M_{P}^{2}}{16 \pi}\left(\frac{1}{r^{2} A B}-\frac{A^{\prime}}{r A^{2} B}-\frac{1}{r^{2} B}\right)= \\
\frac{\alpha}{M_{P}^{2}}\left(1-\frac{2 M}{r M_{P}^{2}}\right)^{-1}\left(\frac{2352\left(M / M_{P}^{2}\right)^{3}}{r^{9}}-\frac{1080\left(M / M_{P}^{2}\right)^{2}}{r^{8}}\right) \\
-\frac{M_{P}^{2}}{16 \pi}\left(\frac{-B^{\prime}}{r A^{2} B}-\frac{1}{r^{2} A^{2}}+\frac{1}{r^{2} A}\right)= \\
\frac{\alpha}{M_{P}^{2}}\left(1-\frac{2 M}{r M_{P}^{2}}\right)\left(\frac{240\left(M / M_{P}^{2}\right)^{3}}{r^{9}}-\frac{216\left(M / M_{P}^{2}\right)^{2}}{r^{8}}\right) \\
\frac{M_{P}^{2}}{16 \pi}\left(\frac{A^{\prime}}{2 r^{3} A^{2}}-\frac{B^{\prime}}{2 r^{3} A B}-\frac{B^{\prime \prime}}{2 r^{2} A B}+\frac{\left(B^{\prime}\right)^{2}}{4 r^{2} A B^{2}}+\frac{A^{\prime} B^{\prime}}{4 r^{2} A^{2} B}\right)= \\
\frac{\alpha}{r^{2} M_{P}^{2}}\left(\frac{-1488\left(M / M_{P}^{2}\right)^{3}}{r^{9}}+\frac{648\left(M / M_{P}^{2}\right)^{2}}{r^{8}}\right)
\end{gathered}
$$


where eq.10 corresponds to the temporal coordinate, eq.11 to the radial one and eq.12 to the angular coordinates (both produce the same equation). Now, from eq.10 we find the coefficients of the $A$ expansion, from eq.11 we find the $B$ coefficients and finally, eq.12 is found to be compatible with the other two. The values found for the coefficients go as follows: $a_{0}=1, a_{1}=$ $2 M / M_{P}, a_{k}=a_{1}^{k}$ for $k=2,3,4,5$ and $a_{k}=a_{1}^{k}+16 \pi \alpha a_{1}^{k-4}(54+(k-6) 5)$ for $k=6,7,8,9$ and $b_{0}=1, b_{1}=-a_{1}, b_{k}=0$ for $k=2,3,4,5,6, b_{7}=-16 \pi \alpha 5 a_{1}^{3}$ and $b_{8}=b_{9}=0$. As expected, the first corrections to the Schwarzschild metric are of the order of $\left(L_{p} / r\right)^{6}$. Note also that now $A$ is not equal to $B^{-1}$ and this fact will have important consequences that will be discussed later. With regard to the validity of the coefficients obtained above, the approximation we have used introducing the Schwarzschild metric in the RHS of eq. 6 gives correct values for the coefficients up to terms of order 9. However, when one consider the effect of possible counterterms in the lagrangian with eight or more derivatives, they could affect coefficients of terms of order seven or higher.

To study the effect of the new $\alpha$ term on the static isotropic solution one could consider an alternative approach by making a perturbative expansion in the $\alpha$ coupling constant instead of an expansion in $L_{p} / r$. To the lowest order, it is possible to find:

$$
\begin{gathered}
A(r)=\frac{1}{1-\frac{2 M}{r M_{P}^{2}}}+16 \pi \alpha\left(\frac{2 M}{M_{P}}\right)^{2}\left(\frac{1}{r M_{P}}\right)^{6}\left(\frac{54}{1-\frac{2 M}{r M_{P}^{2}}}+\frac{10 M /\left(r M_{P}^{2}\right)}{\left(1-\frac{2 M}{r M_{P}^{2}}\right)^{2}}\right) \\
B(r)=1-\frac{2 M}{r M_{P}^{2}}-80 \pi \alpha\left(\frac{2 M}{M_{P}}\right)^{3}\left(\frac{1}{r M_{P}}\right)^{7}
\end{gathered}
$$

It is easy to see that the behaviour of this solution close to the gravitational radius $r_{s}=2 M / M_{P}^{2}$ is now controlled by the $\alpha$ term. For instance, it is now possible to find a value $r_{A}$ near $r_{s}$ where $A\left(r_{A}\right)=0$ but for the ordinary Schwarzschild solution such a point does not exist. In this case the $A$ function diverges and the $B$ function vanishes at the gravitational radius of the body i.e. at the event horizon $A\left(r_{s}\right)=\infty$ and $B\left(r_{s}\right)=0$. Similarly, one could ask where this happens in our modified solution. Then it is not difficult to see that for general $\alpha$ the point $r_{B}$ where $B\left(r_{B}\right)=0$ is different from the point where $A$ diverges which continues being the gravitational radius $A\left(r_{s}\right)=\infty$. For example, for $79|\alpha| \pi\left(M_{P} / M\right)^{4}<<1$ (which is a very good approximation for macroscopic bodies) we find, for positive $\alpha$, that $r_{B}>$ 
$r_{s}>r_{A}$ with $\left.r_{A} \simeq\left(2 M / M_{P}^{2}\right)\right)\left(1-\alpha 5 \pi\left(M_{P} / M\right)^{4}\right)$ and $\left.r_{B} \simeq\left(2 M / M_{P}^{2}\right)\right)(1+$ $\left.\alpha 5 \pi\left(M_{P} / M\right)^{4}\right)$ (where $\mathrm{A}$ vanishes at $r_{A}$ ). For negative $\alpha$ we have $r_{A}>r_{s}>$ $r_{B}$ being $r_{A}$ and $r_{B}$ given by the same equations.

More surprisingly, when the scalar curvature is computed for the modified metric, it is found to be divergent at $r_{A}$ and $r_{B}$. This shows the existence of an intrinsic singularity at least in the most external radius. In addition, the signs of these divergences are different in such a way that in the limit $\alpha$ going

to zero (where the standard Schwarzschild solution must be found) we see that $r_{A}$ and $r_{B}$ go both to $r_{s}$. In this limit the corresponding two divergences of the scalar curvature cancel with each other because of the different sign, the singularity disappear as it must be and the $A=B^{-1}$ relation is recovered.

\section{The modified Robertson-Walker metric:}

The most general isotropic and homogeneous metric can be written as:

$$
d \tau^{2}=d t^{2}-a^{2}(t)\left(\frac{d r^{2}}{1-k r^{2}}+r^{2} d \theta^{2}+r^{2} \sin ^{2} \theta d \phi^{2}\right)
$$

where $k=-1,0,1$ corresponding to an open, flat or closed space and $a(t)$ is the scale parameter which must be determined by the equations of motion. Any isotropic and homogeneous distribution of matter will be described by a energy momentum tensor that takes the form of a perfect fluid:

$$
T^{\mu \nu}=(\rho+p) U^{\mu} U^{\nu}+p g^{\mu \nu}
$$

where $U^{\mu}$ is the velocity four vector, $\rho$ is the density, and $p$ is the pressure. The energy conservation equation reads:

$$
\frac{d\left(\rho a^{3}\right)}{d a}=-3 p a^{2}
$$

and finally, the state equation of matter is needed $(p=p(\rho))$ to specify completely $a(t)$ from the equation of motion and the initial conditions. In the case considered in this paper, the equations of motion are: 


$$
\begin{gathered}
\frac{M_{P}^{2}}{16 \pi}\left(-\frac{3}{a^{2}}\left(a^{\prime 2}+k\right)\right)+\frac{\alpha}{M_{P}^{2}}\left(-24 \frac{a^{\prime \prime 3}}{a^{3}}+12 \frac{\left(a^{\prime 2}+k\right)^{3}}{a^{6}}+\right. \\
\left.72\left(\frac{a^{\prime} a^{\prime \prime} a^{\prime \prime \prime}}{a^{3}}-\frac{a^{\prime 2}\left(a^{\prime 2}+k\right)^{2}}{a^{6}}\right)\right)+\frac{\rho}{2}=0
\end{gathered}
$$

which is the equation corresponding to the temporal coordinate. The three spatial coordinates give rise to only one independent equation.

$$
\begin{array}{r}
\frac{M_{P}^{2}}{16 \pi}\left(\frac{2 a^{\prime \prime}}{a}+\frac{a^{\prime 2}+k}{a^{2}}\right)+\frac{\alpha}{M_{P}^{2}}\left(12 \frac{\left(a^{\prime 2}+k\right)^{3}}{a^{6}}\right. \\
\left.-72\left(\frac{a^{\prime \prime \prime \prime} a^{\prime \prime}+a^{\prime \prime \prime 2}}{3 a^{2}}-\frac{a^{\prime \prime}\left(a^{\prime 2}+k\right)\left(5 a^{\prime 2}+k\right)}{3 a^{5}}+\frac{a^{\prime 2}\left(a^{\prime 2}+k\right)^{2}}{a^{6}}\right)\right)+\frac{p}{2}=0
\end{array}
$$

Using the energy conservation equation it is not difficult to see that the derivative of the first equation above is in fact the addition of the two equations. Therefore, we can take eq.18 as the only independent equation. However, this equation seems to be very difficult to solve. In order to find some exact solutions we have tried to look for $a(t)$ functions which also satisfy the relation

$$
a^{\prime 2}+k=C a^{n}
$$

The eq.18 forces $n$ to be equal 2 and:

$$
\frac{M_{P}^{2}}{16 \pi} C+\frac{4 \alpha}{M_{P}^{2}} C^{3}=\frac{\rho}{6}
$$

Thus $\rho$ must be a constant and this fact, together with the conservation of the energy equation imply the state equation $p=-\rho$. This means that the kind of solutions we are dealing with can describe matter only in the form of a cosmological constant $\rho=\lambda M_{P}^{2} / 8 \pi$. In any case we can use eq.21 to obtain the constant $C$ in terms of $\alpha$ and $\lambda$. Independently of this value,eq.20 can be easily integrated with $n=2$ and the following solutions are obtained:

a) In the case $C=0$ the eq.20 reads $a^{\prime 2}=-k$ so for $k=0 a$ is constant and for $k=-1, a(t)= \pm t+t_{0}$. This case corresponds to the standard Einstein equation with $\alpha=0$ in absence of matter. 
b) For $C>0$ eq.20 reads $a^{\prime 2}=C a^{2}-k$. For $k=1, k=-1$ and $k=0$ the solutions are respectively:

$$
\begin{aligned}
& a(t)=\frac{1}{\sqrt{C}} \cosh \left(\sqrt{C}\left(t-t_{0}\right)\right) \\
& a(t)=\frac{1}{\sqrt{C}} \sinh \left(\sqrt{C}\left(t-t_{0}\right)\right) \\
& a(t)=a\left(t_{0}\right) \exp \left(\sqrt{C}\left(t-t_{0}\right)\right)
\end{aligned}
$$

c) For $C<0$ there is a solution only in the case $k=-1$ where

$$
a(t)=\frac{1}{\sqrt{-C}} \sin \left(\sqrt{-C}\left(t-t_{0}\right)\right)
$$

We would like to stress that all these solutions satisfy exactly eq.18 with the Robertson-Walker metric provided that the $C$ is related to $\alpha$ and $\lambda$ through eq.21 with $\rho=\lambda M_{P}^{2} / 8 \pi$. Therefore now we will write $C$ in terms of the above mentioned constants. In particular we have the important case of no matter and no cosmological term i.e. pure gravity where we have three solutions: $C=0, C= \pm \sqrt{-M_{P}^{4} / 64 \pi \alpha}$. Thus, in this case the $C$ different from zero solutions exist only for negative $\alpha$.

In the case of general $\lambda$ one must consider the discriminant of the eq.21. The sign of this discriminant is in fact determined by $\alpha$ and $\lambda$. In any case, it is always possible to find an analytical formula for the roots of eq.21 in terms of $\alpha$ and $\lambda$.

\section{Conclussions:}

At low-energies gravitation can be described by an effective lagrangian which is an expansion in the number of derivatives over $M_{P}$. The first term of this expansion is the standard Einstein lagrangian but those with higher derivatives have undetermined coefficients that finally could be computable from the underlying theory of gravitation in a similar way as the coefficients of the low-energy effective lagrangian for strong interactions could, at least in principle, be obtained from QCD.

Independently of which are the real values of these coefficients, the consistence of the theory at low energies requires the $\alpha R_{\gamma \delta}^{\alpha \beta} R_{\sigma \rho}^{\gamma \delta} R_{\alpha \beta}^{\sigma \rho}$ term considered in this work to be present in order to make it renormalizable in 
the sense of the phenomenological lagrangians. Then it seems to be interesting to ask about the kind of effects that this term could produce and how it can modify the standard predictions of Einstein gravitation.

In the case of the gravitational field produced by a static and spherical body, we obtain that the modifications to the large distance field are rather weak and completely unobservable. However, in the region close to the Schwarzschild radius, the qualitative behavior of the field changes dramatically since the event horizon is transformed in an extended and spherical singularity. In fact, as was already discovered in [11], this is a very generic effect and it will appear virtually in any modification of the field equations and not only in the one considered in this work. We think that, at least to our knowledge, the physical consequences of this fact have not been yet properly studied in the literature.

In the case of the cosmological solution we have shown that inflationary solutions exist for the modified equations of motion which do not require the somewhat artificial introduction of the inflaton field (this interesting phenomenon was also found in [12] for the case of an extra $R^{2}$ term in the gravitational lagrangian). Of course, in the real case one would need to include the effect of higher order terms in the equation of motion but our simple solutions show that pure gravitation could produce inflation and this could be relevant in the very early history of the universe [13].

\section{Note added}

When this work was completed, we noticed reference [14] where the authors study the effect of the six derivative terms on the Hawking temperature of black holes.

\section{Aknowledgments}

This work has been partially supported by the Ministerio de Educación y Ciencia (Spain)(CICYT AEN90-0034). 


\section{References}

[1] E. Alvarez Rev. of Mod. Phys. 61, 561 (1989)

[2] Superstring theory, M.B. Green, J.H. Schwarz and E. Witten. Cambridge University Press (1987)

[3] S. Weinberg, Physica 96A 327 (1979)

[4] J. Gasser and H. Leutwyler, Ann. of Phys. 158142 (1984), Nucl. Phys. B250 465 and 517 (1985)

[5] A. Dobado and M.J. Herrero, Phys. Lett. B228 495 (1989) and B233 505 (1989)

J. Donoghue and C. Ramirez, Phys. Lett. B234 361 (1990)

[6] N. Nakanishi and I. Ojima, Phys. Rev. Lett. 4391 (1979)

[7] G. 't Hooft and M. Veltman, Ann. Inst. H. Poincaré 2069 (1974)

[8] M.H. Goroff and A. Sagnotti, Nucl. Phys. B266 709 (1986)

[9] D.M. Capper, J.J. Dulwich and M. Ramón Medrano, Nucl. Phys. B254 737 (1985)

[10] Gravitation and Cosmology, S. Weinberg, John Wiley \& Sons (1972)

[11] W. Israel, Phys. Rev. 1641776 (1967) 200

[12] A.A. Starobinsky, Phys. Lett. 91B 99 (1980)

[13] A. Dobado and A. López, in preparation.

[14] M. Lu and M.B. Wise, Phys. Rev. D47 3095 (1993) 